Reviews in Digital Humanities • Vol. 1, No. 10

\title{
Review: Marianne Moore Digital Archive
}

\author{
Ashley Palmer ${ }^{1}$ \\ ${ }^{1}$ The University of Alabama
}

Published on: Oct 05, 2020

DOI: $10.21428 / 3 e 88 f 64 f .17 c 8 c d 0 a$

License: Creative Commons Attribution 4.0 International License (CC-BY 4.0). 


\section{Project}

Review: Marianne Moore Digital Archive

\section{Project Director}

Cristanne Miller, University at Buffalo SUNY

\section{Project URL}

http://moorearchive.org $/$

\section{Project Reviewer}

Ashley_Palmer, The University of Alabama

\section{Project Overview}

\section{Cristanne Miller}

The Marianne Moore Digital Archive (MMDA) aims to publish all 120 of Moore's working notebooks, in linked dual-page facsimile and textual editions, to its free public website. Moore kept notebooks on every aspect of her life, from 1905 through 1970recording conversations she overheard among famous peers; notes on her reading and on the lectures, sermons, exhibits, and concerts she attended; and records of her travel and finances. Six notebooks contain early drafts of poems. The MMDA has published three notebooks and hopes to publish another two by December 2020. These notebooks have never been published and are virtually inaccessible, since they are held physically at the Rosenbach Museum \& Library in Philadelphia, PA, which is open to scholars only 18 hours a week.

The MMDA is both a research and an educational site for an audience of scholars, teachers, students, and lay readers interested in modernism. Our transcriptions transform the possibilities of Moore studies and broader work on modernist poetry and the intersections of daily life and the arts in the early 20th century. Our transcriptions prevent laborious deciphering of handwriting; our annotations clarify Moore's engagement with 20th-century culture and politics and identify the publications she quotes from or refers to. The site also includes hard-to-find scholarly essays, a bibliography, syllabuses and lesson plans, interpretive readings of poems, a timeline, and other features. "Submit" buttons enable contributions to the site. We are adding "comment" buttons to enable suggestions for improvement or correction. 
After obtaining exclusive permission from the Moore Estate to publish these materials, Cristanne Miller applied for a University at Buffalo SUNY (UB) start-up grant, collaborating with Srirangaraj Setlur and Ed Sobczak. Nikolaus Wasmoen joined her as technical director and Robin Schulze, Elizabeth Gregory, and Heather White serve as associate directors/editors. This team developed the MMDA web platform (using HubZero 2.0) to enable multiple project collaborators to transcribe, proof, and encode within a single environment that securely houses all image and TEI files, documentation, internal project Wiki, and other resources. The MMDA has a customized, TEI P5-compliant RelaxNG encoding schema and uses XML/TEI encoding for notebook editions (see Project Documentation). All editors are Moore scholars, Wasmoen has national expertise as a digital humanities technical director, and Sobczak continues to assist with web programming. All materials are backed up to the highest security and data protection standards.

The MMDA has been exhibited as display or through presentations at Modernist Studies Association Conferences since 2015, and Miller and Wasmoen have published and presented on the site in Canada, the U.S., and the Netherlands. We are responding to peer review for Spring 2020 aggregation with ModNets. We have applied for several grants and are awaiting word from the NEH and ACLS. Current funding comes from a small endowment created by Moore scholars, Miller's research budget, and small grant assistance (\$1-5000) from various sources at UB. Only four years old, the site is already being cited in publications and presentations and used in classrooms, from Buffalo to Europe, China, and Brazil.

\section{Project Review}

\section{Ashley Palmer}

The meticulous and visually appealing Marianne Moore Digital Archive (MMDA) has a lot to offer scholars of Moore's work, teachers of modernism and American literature, and visitors with a general interest in modernism. As a contribution to scholarly study, the MMDA presents digital editions of Moore's working notebooks, annotated and transcribed by the MMDA team. The site currently has 3 notebooks completed with more forthcoming, and they aim to eventually feature all 120 of Moore's notebooks. Beyond these richly detailed notebooks, the MMDA also contains contextual materials that would interest long-time scholars as well as newcomers looking for an introduction to Moore. Additional highlights include Moore's Newsletters (complete with engaging animal sketches), links to full-text scholarship on Moore's work, a 
timeline of her life and publications, critical excerpts about her major poems, and resources for teaching Moore in the classroom. The promise of a forthcoming glossary (under development) and the additional notebook transcriptions encourages visitors to check back for new content.

The expanded access the MMDA affords to Moore's archive is impressive. Not only does the site make her fragile and difficult-to-access notebooks (housed in the Rosenbach Museum in Philadelphia, PA) widely available as digital images, but it also presents transcriptions of Moore's handwriting alongside those facsimiles, deciphering Moore's cursive to ease the viewer's access to her words. Annotations included within the transcriptions clarify obscure references and illuminate intertextual connections. The expertise evident in these annotations can be traced back to the Moore scholars at the helm of the MMDA, led by director Cristanne Miller.

The three published notebook transcriptions show the promise of this ambitious project, which has much more room to expand. It's understandable that the process would take time, given how thoroughly curated the notebooks are: not only are they digitized, transcribed, and annotated, but they are also accompanied by introductions that summarize and contextualize each notebook's content. Additionally, the site includes supplementary texts that are referenced within Moore's notebooks. The effect is something akin to a critical edition in website format, and it picks up Stephen Ross and Jentery Sayers' call to "highlight the relationship between individual works and the cultures in which they were embedded" (625).

The notebook transcriptions utilize TEI (Text Encoding Initiative) and customized EVT (Edition Visualization Technology) version 1.0, developed at the University of Buffalo's Center for Unified Biometrics and Sensors. This technology enables some fun navigation options, including different tags for verse and prose writing and synced facsimile-transcription highlighting that simultaneously highlights small clusters of Moore's handwriting alongside their corresponding typed transcription. One thing this encoding should enable, which I would love to see in the future, is a way to fully search all of the language of the transcriptions and the annotations that accompany them (the Newsletter and other pages on the website are fully searchable). Although it seems likely that the forthcoming glossary will guide users to proper names of people, characters, organizations, and institutions by directing them to the appropriate notebook where they appear, a search covering all of the language of the transcriptions and annotations across the notebooks would support even more targeted queries. 
A collaborative effort between multiple universities and fields of study, the MMDA expands access to Moore's work in many ways: presenting clear and elucidating access to her notebooks, linking from the notebooks to contexts that shed light on her network of influences, incorporating scholarship on Moore's writing, supplying lesson plans that can steer new groups of students, and soliciting ongoing contributions by scholars. If the work of modernist digital humanities is to "analyze modernism as a set of material, institutional, and collaborative practices" (Ross), then the MMDA excels at this inclusive and complex project, which should inspire and support new ways of studying Marianne Moore.

Works Cited

Ross, Shawna. "From Practice to Theory: A Forum on the Future of Modernist Digital Humanities." Modernism/Modernity, vol. 3, no. 2 [Online section], July

2018. EBSCOhost, search.ebscohost.com/login.aspx?

direct $=$ true $\& d b=\operatorname{mlf} \& A N=2018402358 \&$ site $=$ ehost-live\&scope $=$ site .

Stephen Ross and Jentery Sayers, “Modernism Meets Digital Humanities.” Literature Compass, vol. 11, no. 9, 2014, pp. 625-33.

Note: Ashley Palmer completed this review prior to her employment at The University of Alabama, where MMDA Associate Editor Heather Cass White is employed. The editors confirm there was no conflict of interest at the time of the review. 